\title{
Advances in the general factor of personality dynamics
}

\author{
Joan C. Micó \\ Intitut Universitari de Matemàtica Multidisciplinar \\ Universitat Politècnica de València \\ Valencia, Spain \\ jmico@mat.upv.es
}

\author{
Salvador Amigó. \\ Departament de Personalitat, Avaluació i \\ Tractaments Psicològics. \\ Universitat de València \\ Valencia, Spain \\ Salvador.Amigo@uv.es
}

\author{
Antonio Caselles. \\ IASCYS member \\ Departament de Matemàtica Aplicada. \\ Universitat de València \\ Valencia, Spain \\ Antonio.Caselles@uv.es
}

\begin{abstract}
This paper presents a dynamical integro-differential equation to reproduce the dynamical response of the General Factor of Personality (GFP) to a stimulus dose, particularly to a stimulant drug dose. The model is called in the past authors publications as response model. We refer to it as the old response model, due to a new response model presented here that solves partially the problem of the model validation: how to forecast the GFP dynamical response from a previous model calibration. The application case presented is an individual ABC experimental design where the stimulus used is methylphenidate.
\end{abstract}

Keywords: General Factor of Personality; dynamics; integro-differential equation; calibration; validation; methylphenidate.

\section{Introduction}

The aim of this paper is to present the advances deduced from new research about the General Factor of Personality (GFP) dynamics performed with the response model.

The GFP is a trait of human personality defined for the first time by Salvador Amigó [1]. It asserts for a single trait, the GFP, to describe the overall personality, which has a biological base inside the general activation of the stress system. A questionnaire to measure the individual GFP is provided in [2]. The dynamical response of the GFP to a stimulus can be described by the response model: a dynamical integro-differential equation. The response model has been evaluated in different works by using different drug stimulus, such as caffeine or methylphenidate [3, 4, 5, 6, 7]. A list of 5 adjectives was developed to measure the dynamical change of the GFP [8]. The results of this questionnaire correlate positively with the questionnaire provided in [2]. In addition, it is used in this paper in the context of the application case to measure the individual GFP dynamical change.

The advances presented on the GFP dynamics are provided by a new response model, which has two novelties respect the old one: (1) the term of the new response model called as excitation effect becomes nonlinear; (2) the parameter called as tonic level acts in a different mathematical way in the new response model. These changes can be considered advances because, on a hand, the nonlinear term provides a more realistic dynamics for the stimulus and, on the other hand, the change in the tonic level, maintaining constant the rest of the parameter values, provides a better way to validate the response model.

These advances are implemented on part of the ABC experimental design presented in [5] as application case. The part considered is that devoted to the GFP measured by the five adjectives scale: a basic line (A), a subsequent individual consumption of $20 \mathrm{mg}$ of methylphenidate (B), and a subsequent consumption of $40 \mathrm{mg}$ of methylphenidate (C). The results of the response model calibration are presented for both the old response model and the new response model. The differences are then stressed in favour of the new response model. In addition, a new way to validate de model is also provided.

Section 2 is devoted to present the old response 
model and the changes that provide the new one, as well as the hypotheses under which these changes are done. Section 3 is devoted to the calibration of the old response model, while Section 4 is devoted to the validation of the new response model, as well as the obtained advances. Section 5 is devoted to the paper conclusions. After the section References, the section Appendix is devoted to present figures and tables.

\section{The old and new response models}

The kinetic part of the old response model provides the evolution of the stimulus amount in organism after being consumed. It is given by two coupled differential equations:

$$
\left.\begin{array}{c}
\frac{d m(t)}{d t}=-\alpha \cdot m(t) \\
m(0)=M
\end{array}\right\}
$$

In (1) $m(t)$ is the non-assimilated stimulus amount, $M$ is the initial amount of a drug single dose, and $\alpha$ is the stimulus assimilation rate. In (2) $s(t)$ represents the stimulus, i.e., the amount of stimulus in the organism not yet consumed (or metabolized) by cells, $s_{0}$ is the amount of stimulus present in the organism before the dose intake, and $\beta$ is the stimulus elimination rate. Integrating the system (1)-(2), the analytical solution of the stimulus as a function of time $t$ and the referred parameters is obtained:

$$
s(t)=\left\{\begin{array}{c}
\frac{\alpha \cdot M}{\beta-\alpha}(\exp (-\alpha \cdot t)-\exp (-\beta \cdot t)): \alpha \neq \beta \\
\alpha \cdot M \cdot t \cdot \exp (-\alpha \cdot t): \alpha=\beta
\end{array}\right.
$$

Equation (3) assumes that $s_{0}=0$, i.e., no stimulus is present in the organism before the experiment.

The dynamics of the GFP is given by the following equation:

$$
\left.\begin{array}{c}
\frac{d y(t)}{d t}=a(b-y(t))+\frac{p}{b} s(t)- \\
-b \cdot q \cdot \int_{0}^{t} \mathrm{e}^{\frac{x-t}{\tau}} \cdot s(x) \cdot y(x) d x \\
y(0)=y_{0}
\end{array}\right\}
$$

In (4), $s(t)$ represents the stimulus given by (3); $y(t)$ represents the GFP dynamics; and $b$ and $y_{0}$ are respectively its tonic level and its initial value.

The dynamics of (4) is a balance of three terms, which provide the time derivative of the GFP: the homeostatic control $a(b-y(t))$, i.e., the cause of the fast recovering of the tonic level $b$, the excitation effect $(p$. $s(t) / b)$, which tends to increase the GFP, and the inhibitor effect $\left(b \cdot q \cdot \int_{0}^{t} \mathrm{e}^{\frac{x-t}{\tau}} \cdot s(x) \cdot y(x) d x\right)$, which tends to decrease the GFP and is the cause of a continuously delayed recovering, with the weight $\mathrm{e}^{\frac{x-t}{\tau}}$. Parameters $a, p, q$ and $\tau$ are named respectively the homeostatic control power, the excitation effect power, the inhibitor effect power and the inhibitor effect delay. All the parameters of the model depend on the individual personality or individual biology and on the type of stimulus. The correct interpretation of the tonic level $b$ is important to be stressed: its value is situational and depends on the individual and the kind of stimulus.

In conclusion, Equations (3) and (4) define the old response model. The proposed changes on this model are referred to (4), i.e.:

$$
\left.\begin{array}{c}
\frac{d y(t)}{d t}=a(b-y(t))+\frac{p}{b} s(t) \cdot y(t)- \\
-\frac{q}{b} \int_{0}^{t} \mathrm{e}^{\frac{x-t}{\tau}} \cdot s(x) \cdot y(x) d x \\
y(0)=y_{0}
\end{array}\right\}
$$

Eqs. (3) and (5) define the new response model. Note in (5) that the excitation effect becomes now nonlinear $\left(\frac{p}{b} s(t) \cdot y(t)\right)$. This hypothesis tries to reproduce in a more realistic way the fact that, in the sensitization process the more an individual consumes a drug the more he/she wishes the drug. In fact, the inhibitor effect assumes a similar role in the habituation process [1]. In addition, the other hypothesis assumes that the tonic level divides both effects. This assumption is due to the more extraverted individuals perceive of a stronger manner both effects and vice versa [1].

\section{Calibration of the old response model}

The necessary figures and tables are presented in the Appendix at the end of the paper. Note that the calibrations of the old and new response models do not fit the amount of the methylphenidate dose because they are provided to the individual with determined values: $M=20 \mathrm{mg}$ for Phase B, and $M=40 \mathrm{mg}$ for Phase C.

First of all the results of Phase A are presented in Fig. 1. Note that Phase A works correctly as a control phase: the GFP values oscillate inside a small interval around a mean value, the initial condition excepted. The comparatively great value of the initial condition can be explained for the expectation created in the individual by the experimental design.

The calibration of the old response model corresponding to Phase B is presented in Fig. 2, with a determination coefficient $\mathrm{R}^{2}=0.96$ and random residuals. The corresponding optimal parameter values are presented in Table 1. It may be observed that these parameter values do not fit Phase C. Even arbitrary subsets of these parameter values neither fit Phase C. Thus, Phase C needs an independent calibration, which is presented in Fig. 3, with a determination coefficient $\mathrm{R}^{2}=0.83$ and random residuals. The corresponding optimal parameter values are presented in Table 2 .

The conclusion obtained from these facts is that the old response model can be calibrated, but it does not resist a validation process, which would imply the use of the optimal parameter values of Phase B to reproduce Phase $\mathrm{C}$ with a high determination coefficient and random residuals.

In addition, the stimulus dynamics (the amount of 
methylphenidate in organism) presents non-realistic patterns, represented in Fig. 4 for Phase B and in Fig. 5 for Phase C. In both patterns the amount of methylphenidate is still increasing as the effect on the GFP has almost vanished (see Figs. 1 and 2). This fact is also an important validation objection for the old response model. A realistic pattern would be the practical vanishing of the stimulus after the four hours of both experiments.

Thus, the new response model presented in the next section is the tool considered to try to solve these validation problems.

\section{Validation of the new response model}

The new response model (Eqs. 3 and 5) can be validated in the context of the ABC experimental design in the following terms.

Phase $\mathrm{A}$ is the same than that of the old response model (Fig. 1). Thus, the new response model is calibrated for Phase $\mathrm{B}$, and the corresponding optimal parameter values are obtained. These values are used to reproduce the dynamics of Phase $C$, with the exception of the tonic level, which is re-calibrated. Fig. 6 (Phase B) and Fig. 7 (Phase $C$ ) shows that the new response model can be calibrated for Phase B and subsequently validated for Phase $\mathrm{C}$, with the only change of the tonic level. In fact, the determination coefficients are very high and similar to those of the old response model $\left(\mathrm{R}^{2}=0.96\right.$ for Phase $B$ and $R^{2}=0.76$ for Phase $C$ ), with random residuals. Table 3 provides the optimal parameter values for both phases.

In addition, note that the stimuli dynamics corresponding to both phases have the same parameter values ( $\alpha$ or stimulus assimilation rate and $\beta$ or the stimulus elimination rate), thus the stimulus of Phase $C$ is double of the one of Phase $B$ due to the double methylphenidate dose provided in Phase $\mathrm{C}$ respect to Phase B. The stimulus dynamics of Phases B and C are represented, respectively, in Fig. 8 and Fig. 9. The important feature of these stimuli is that both represent realistic patterns, due to they practically vanish after the four hours of the experiment duration period.

This last shared pattern of both stimuli, and the possibility of the model validation by only changing the tonic level in Phase $C$ respect to Phase $B$, support the convenience to use the new response model as a mathematical tool to reproduce the dynamical response of the GFP to a drug dose consumption, particularly to a methylphenidate dose consumption, instead of the old response model.

\section{Conclusions}

The main conclusion of this paper is that the new response model solves the problem of the dynamical response validation with the re-calibration of an only parameter value. In fact, in the context of the experimental design here presented, the old response model can only be used to calibrate it inside each phase. It implies that once calibrated (Phase B), the old response model can inform nothing about a future time (Phase C), and it must be calibrated again. In addition the calibration of the stimulus presents non-realistic patterns, i.e., the non-vanishing stimulus amount in the organism after the experiment duration period.
However, the new response model, also in the context of the experimental design here presented, can be validated with: (a) its previous calibration in the first dynamical response (Phase B); (b) its subsequent reproduction of a future dynamical response (Phase $\mathrm{C}$ ) by the only re-calibration of the tonic level. In addition the calibration of the stimulus presents realistic patterns, i.e., the vanishing stimulus amount after the experiment duration period holds.

Nevertheless, actually the re-calibration of the tonic level in Phase $\mathrm{C}$ provides only a partial validation. New investigations should provide the way to validate the new response model with no new recalibrations.

A metaphor thought to investigate the answer to the presented problem may be the following. Imagine that the dynamical response to a stimulus is similar to the electron state in a hydrogen atom in a determined energy level, for instance, the basic level for the principal quantum number $n=1$. This state is mathematically a space distribution with a known probability. Due to a stimulus (in the metaphor, a photon radiation), the electron can jump to an excited energy level, for instance, the second principal quantum number $n=2$. In the new state the space distribution is different and can be known in advance by the quantum theory.

However, the necessary additional theory, equivalent to the quantum theory, is not known in the context of the new response model. Nevertheless, a hypothesis can be stated: similarly to the energy levels of a hydrogen atom, the tonic level values can change depending on some quantum numbers. But this hypothesis implies as well the existence of quantum dynamical responses, and to investigate the reach of this hypothesis, a spatio-temporal approach must be stated for the dynamical responses, similar to the one presented in [9]. The authors think that a spatio-temporal approach can provide the quantization of the tonic level, knowing in advance its possible values. The investigation is thus open.

\section{REFERENCES}

[1] S. Amigó, La teoría del rasgo único de personalidad. Hacia una teoría unificada del cerebro y la conducta (The unique-trait personality theory. Towards a unified theory of brain and conduct). Editorial de la Universidad Politécnica de Valencia, 2005.

[2] S. Amigó, A. Caselles \& J. C. Micó, “The General Factor of Personality Questionnaire (GFPQ): Only one factor to understand the personality?”, The Spanish Journal of Psychology, vol. 13 (2010), Issue 1, pp. 5-17.

[3] S. Amigó, A. Caselles \& J. C. Micó , “A dynamic extraversion model: the brain's response to a single dose of a stimulant drug", British Journal of Mathematical and Statistical Psychology, vol. 61 (2008), pp. 211-231.

[4] A. Caselles,J. C. Micó \& S. Amigó, “ Dynamics of the General Factor of Personality in response to a single dose of caffeine”, The Spanish Journal of Psychology, vol. 14 (2011), pp. 675-692.

[5] J.C. Micó, S. Amigó \& A. Caselles, (2012). “Changing the General Factor of Personality and the c-fos expression with methylphenidate and Self-Regulation Therapy”, The Spanish Journal of Psychology, vol. 15 (2012), pp. 850-867.

[6] J. C. Micó, A. Caselles, S. Amigó, A. Cotolí \& M. T. Sanz, “A mathematical approach to the body-mind problem from a System Personality theory", Systems Research and Behavioral Science, vol. 30 (2013), pp. 735-749.

[7] J. C. Micó, S. Amigó \& A. Caselles, "From the Big Five to the General Factor of Personality: a Dynamic Approach”, The Spanish Journal of Psychology, vol. 17 (2014), pp. 1-18. 
[8] S. Amigó, J. C. Micó \& A. Caselles, "Five adjectives to explain the whole personality: a brief scale of personality”, Revista Internacional de Sistemas, vol. 16 (2009), pp. 41-43. (Available in http://www.uv.es/caselles/).

[9] J. C. Micó \& M. T. Sanz, “A spatio-temporal approach to brain dynamics”, Revista Internacional de Sistemas, vol. 18 (2013), pp. 29-38 (Available in http://www.uv.es/caselles/).

\section{Appendix}

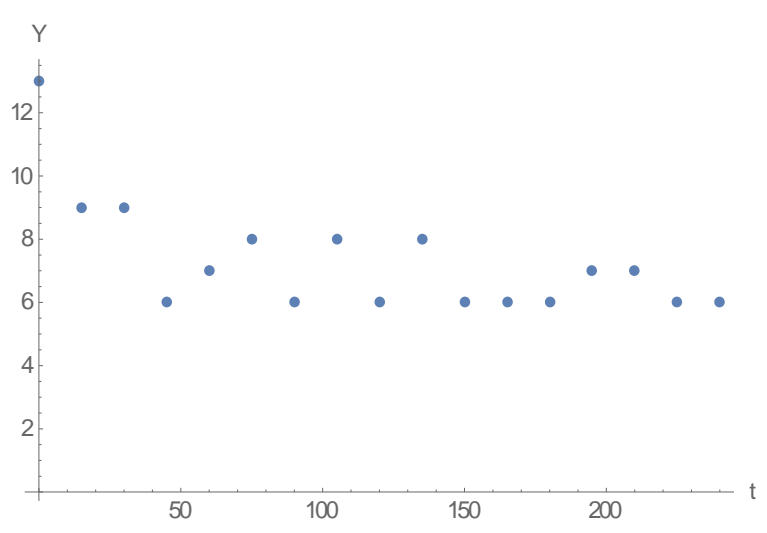

Fig. 1: GFP (Y) versus time (t) in Phase A

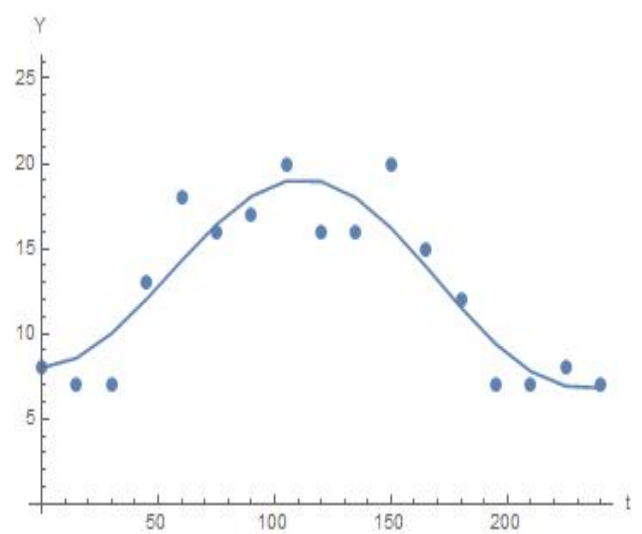

Fig. 2: GFP $(Y)$ versus time $(t)$ in Phase B fitted by the old response model. $\mathrm{R}^{2}=0.96$.

Table 1: optimal values of the old response model parameters for Phase B, corresponding to the GFP dynamics (Y).

\begin{tabular}{ccc}
$\begin{array}{c}\text { Parameter } \\
\text { symbol }\end{array}$ & Name & Optimal value \\
\hline$M$ & $\begin{array}{c}\text { Methylphenidate } \\
\text { dose }\end{array}$ & 20.0 \\
\hline$\tau$ & $\begin{array}{c}\text { Inhibitor effect } \\
\text { delay }\end{array}$ & 154.8481019072612500 \\
\hline$\alpha$ & Assimilation rate & 0.0008391856122216 \\
\hline$\beta$ & Elimination rate & 0.0003031001785003 \\
\hline$a$ & $\begin{array}{c}\text { Homeostatic } \\
\text { control power }\end{array}$ & 0.0000865266711130 \\
\hline$p$ & Tonic level & 14.6301269531250000 \\
\hline$p$ & $\begin{array}{c}\text { Excitation effect } \\
\text { power }\end{array}$ & 4.0442358031868935 \\
\hline & Inhibitor effect & 0.0000161711124331 \\
& power &
\end{tabular}

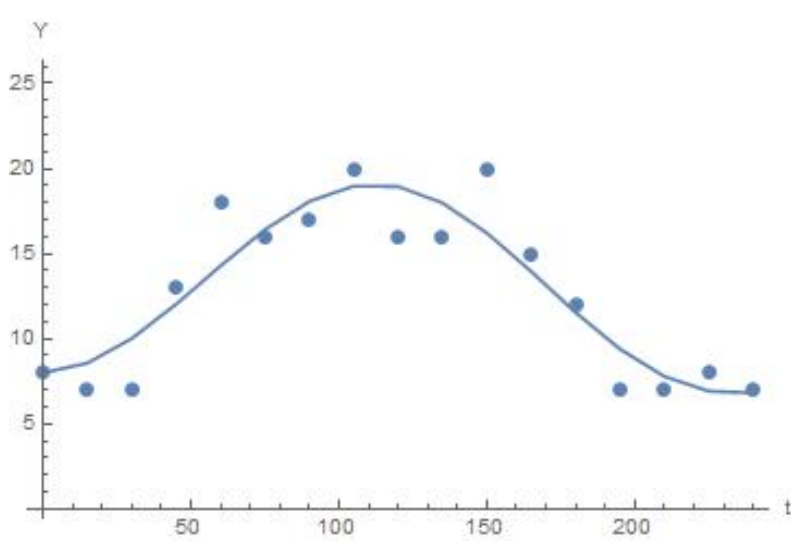

Fig. 3: GFP $(Y)$ versus time $(t)$ in Phase $C$ fitted by the old response model. $\mathrm{R}^{2}=0.83$.

Table 2: optimal values of the old response model parameters for Phase C, corresponding to the GFP dynamics $(Y)$.

\begin{tabular}{ccc}
$\begin{array}{c}\text { Parameter } \\
\text { symbol }\end{array}$ & Name & Optimal value \\
\hline$M$ & $\begin{array}{c}\text { Methylphenidate } \\
\text { dose }\end{array}$ & 40.0 \\
\hline$\tau$ & $\begin{array}{c}\text { Inhibitor effect } \\
\text { delay }\end{array}$ & 78.9311993122100830 \\
\hline$\alpha$ & Assimilation rate & 0.0012873707711697 \\
\hline$a$ & Elimination rate & 0.0035044766097142 \\
\hline & Homeostatic & 0.0005049673774340 \\
\hline
\end{tabular}




\begin{tabular}{ccc}
\hline \multicolumn{3}{c}{ control power } \\
\hline$b$ & Tonic level & 13.4796142578125000 \\
\hline$p$ & $\begin{array}{c}\text { Excitation effect } \\
\text { power }\end{array}$ & 1.3499921431412076 \\
\hline$q$ & $\begin{array}{c}\text { Inhibitor effect } \\
\text { power }\end{array}$ & 0.0000112408190355 \\
\hline
\end{tabular}

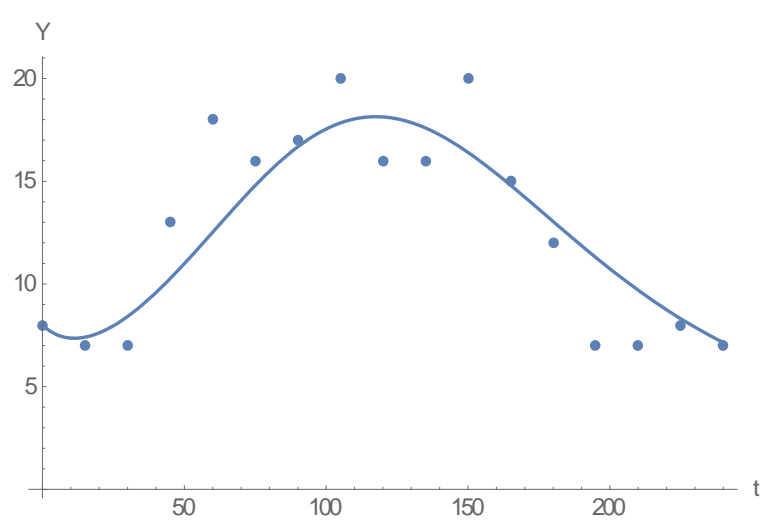

Fig. 7: GFP $(Y)$ versus time $(t)$ in Phase $C$ fitted by the new response model. $\mathrm{R}^{2}=0.76$.

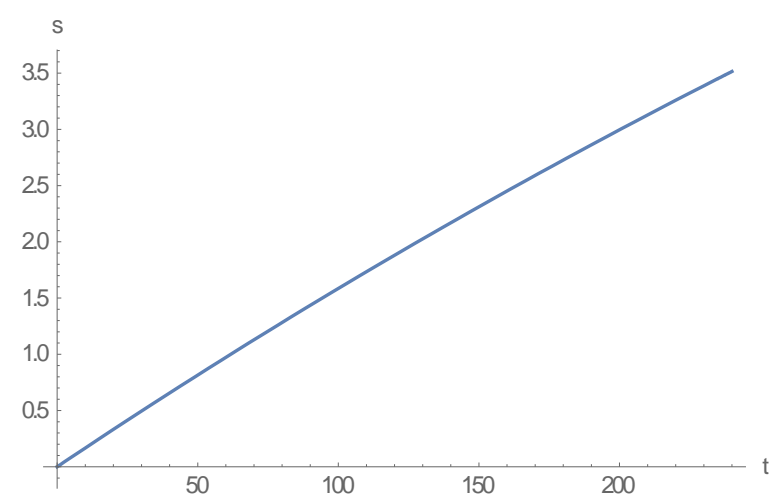

Fig. 4: Stimulus or amount of methylphenidate (s) versus time $(t)$ in organism in Phase B fitted by the old response model.

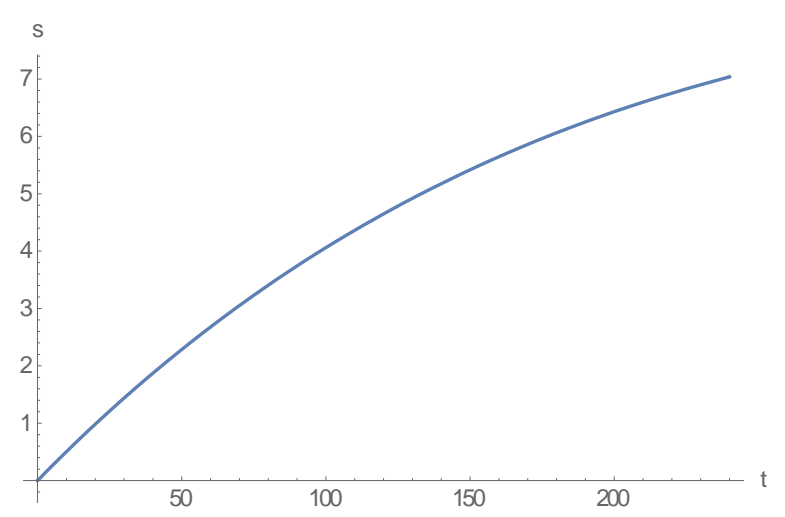

Fig. 5: Stimulus or amount of methylphenidate (s) versus time $(t)$ in organism in Phase C fitted by the old response model.

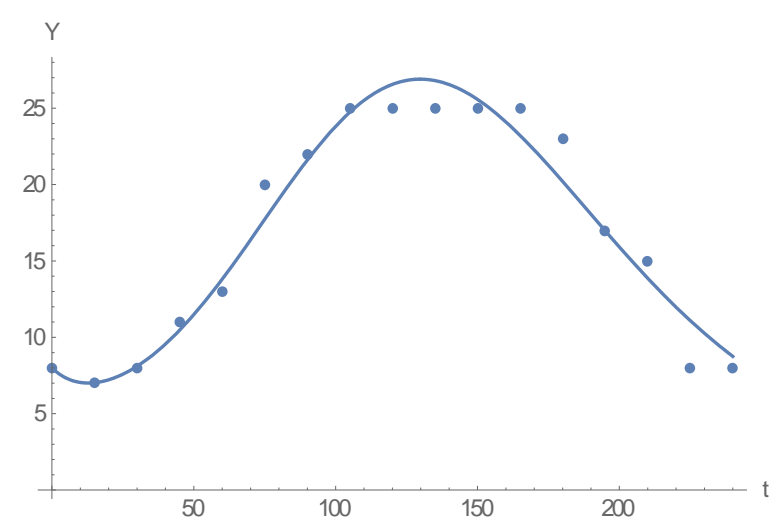

Fig. 6: GFP $(Y)$ versus time $(t)$ in Phase B fitted by the new response model. $\mathrm{R}^{2}=0.96$.
Table 3: optimal values of the new response model parameters for Phases B and C, corresponding to the GFP dynamics ( $Y$ ).

$\begin{gathered}\text { Parameter } \\ \text { symbol }\end{gathered}$ Name

\begin{tabular}{ccc}
\hline$M$ & $\begin{array}{c}\text { Methylphenidate } \\
\text { dose }\end{array}$ & $\begin{array}{c}20.0 \text { (Phase B) } \\
40.0 \text { (Phase C) }\end{array}$ \\
\hline$\tau$ & $\begin{array}{c}\text { Inhibitor effect } \\
\text { delay }\end{array}$ & 1.7947700937950430 \\
$\alpha \alpha$ & Assimilation rate & 0.0260413702093316 \\
\hline$\beta$ & Elimination rate & 0.0130043770613345 \\
\hline$a$ & Homeostatic & 0.0269896307547583 \\
& control power & 1.1960178709357143 \\
(Phase B) & 3.1104237023479389 \\
& Tonic level & (Phase C) \\
\hline
\end{tabular}




\begin{tabular}{ccc}
\hline$p$ & $\begin{array}{c}\text { Excitation effect } \\
\text { power }\end{array}$ & 0.0053418060584095 \\
\hline$q$ & $\begin{array}{c}\text { Inhibitor effect } \\
\text { power }\end{array}$ & 0.0001222167981174 \\
\hline
\end{tabular}

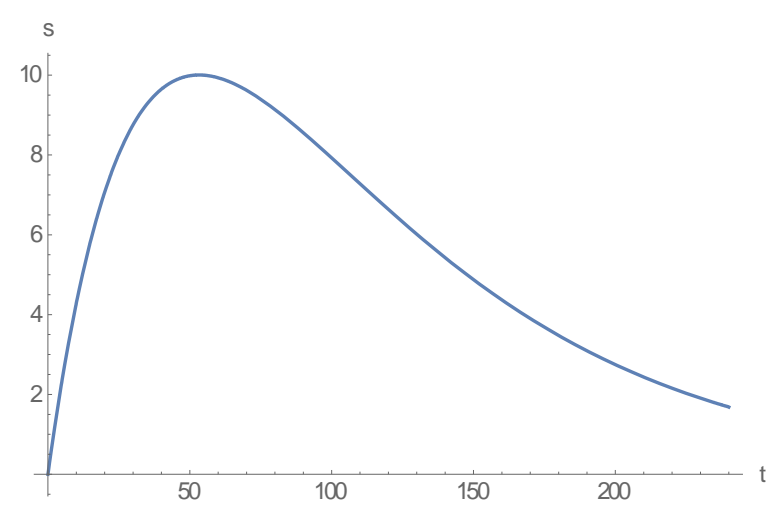

Fig. 8: Stimulus or amount of methylphenidate (s) versus time $(t)$ in organism in Phase B fitted by the new response model.

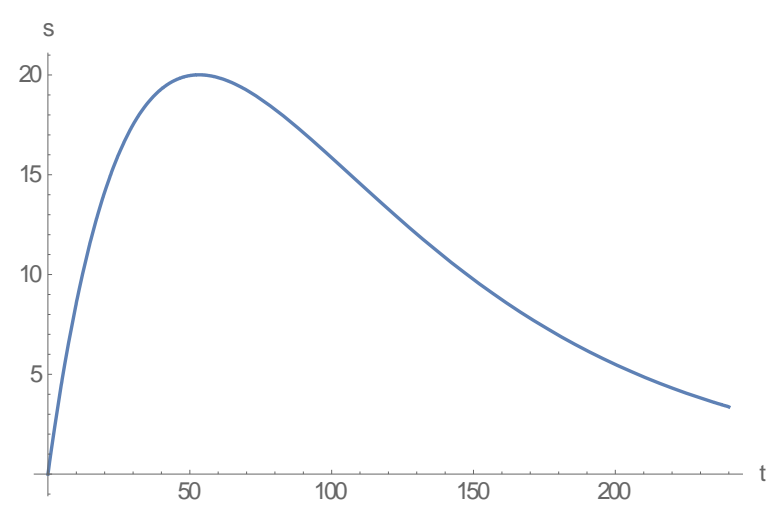

Fig. 9: Stimulus or amount of methylphenidate (s) versus time $(t)$ in organism in Phase $\mathrm{C}$ fitted by the new response model. 